\title{
Microvascular density and vascular endothelial growth factor and osteopontin expression during the implantation window in a controlled ovarian hyperstimulation rat model
}

\author{
XIN GONG $^{1}$, QING TONG ${ }^{1}$, ZHENZHEN CHEN $^{2}$, YUNNA ZHANG ${ }^{1}$, CAI XU ${ }^{1}$ and ZHE JIN ${ }^{1}$ \\ ${ }^{1}$ Reproductive Endocrinology Center, Dongfang Hospital of Beijing University of Chinese Medicine, Beijing 100078; \\ ${ }^{2}$ School of Chinese Materia Medica, Beijing University of Chinese Medicine, Beijing 100102, P.R. China
}

Received May 19, 2014; Accepted December 11, 2014

DOI: $10.3892 /$ etm.2015.2181

\begin{abstract}
Vascular endothelial growth factor (VEGF) and osteopontin (OPN) are suggested to facilitate angiogenesis and vascular remodeling in endometrial receptivity. Determination of the endometrial microvascular density (MVD) is the commonest method used to indirectly assess the levels of vasculogenesis and angiogenesis; however, the associations among VEGF, OPN and MVD remain unclear. Controlled ovarian hyperstimulation $(\mathrm{COH})$ with the gonadotrophin-releasing hormone agonist-long protocol may impair endometrial receptivity, and Traditional Chinese Medicine (TCM) may exert therapeutic effects to relieve this impairment. The aim of the present study was to investigate the effects of $\mathrm{COH}$ on implantation biology and pregnancy outcome, and to explore the potential therapeutic role of the TCM Zi Dan Yin (ZDY). Female Sprague Dawley rats were divided into four groups: Control, $\mathrm{COH}, \mathrm{ZDY}$ and $\mathrm{COH}+\mathrm{ZDY}$. On days 3, 4 and 5 of pregnancy (D3, D4 and D5, respectively), endometrial MVD was measured with cluster of differentiation 34 immunohistochemical detection, and VEGF and OPN protein and mRNA expression was detected through western blotting and reverse transcription-quantitative polymerase chain reaction (RT-qPCR) analysis. On D10, the average number of implantation sites was observed. Subsequent to conceiving and bearing newborn rats, the number of live births from each group was recorded. $\mathrm{COH}$ was shown to have adverse effects on implantation and pregnancy outcome. The MVD was found to be significantly increased in the $\mathrm{COH}$ group compared with that in the control, ZDY and COH+ZDY groups. The results of the protein and RT-qPCR analysis of VEGF and OPN revealed the same trend. Conversely, ZDY reversed the changes in
\end{abstract}

Correspondence to: Professor Zhe Jin, Reproductive Endocrinology Center, Dongfang Hospital of Beijing University of Chinese Medicine, 6 Fangxingyuan 1 Qu, Fengtai, Beijing 100078, P.R. China

E-mail: zjin0129@163.com

Key words: angiogenesis, adhesion, animal model, Chinese herbs, receptivity endometrial MVD, VEGF and OPN, and was indicated to improve uterine receptivity and pregnancy outcome. No significant difference was observed among the control, ZDY and $\mathrm{COH}+\mathrm{ZDY}$ groups. In conclusion, since the results for MVD and VEGF and OPN expression were consistent, MVD could be used as an alternative approach to identify the period of receptivity in rats.

\section{Introduction}

The implantation of a mammalian embryo is a crucial step in the establishment of a normal pregnancy. To prepare for implantation, the uterus undergoes dynamic and tightly regulated proliferation and differentiation following stimulation by changes in the levels of the ovarian hormones estrogen and progesterone (1). Implantation comprises three stages: Apposition, adhesion and penetration. In the apposition stage, the blastocyst unstably adheres to the endometrial surface (2). Following apposition, the trophoblast and luminal epithelium adhere sufficiently strongly to prevent the displacement of the blastocyst (3). The embryo then invades through the luminal epithelium into the stroma to associate with the maternal vasculature (4).

In rats, implantation occurs at a specific time, during a brief $24-\mathrm{h}$ period 5 days after mating $(5,6)$. Prior to implantation, the endometrium is unresponsive to the blastocyst or to other decidualizing signals. In mammals, implantation is regulated through the normal reproductive cycle and, in numerous mammalian species, may be timed to coincide with conditions that are more favorable for the support of embryonic growth (7). The control of implantation is primarily maternal and achieved through hormone-mediated changes in the expression of adhesion molecules and vessel-related factors.

Osteopontin (OPN) is an adhesion protein (8). Amino acid analysis of OPN has revealed the existence of a conserved cell-binding arginine-glycine-aspartic acid sequence, which has been demonstrated to be involved in adherence to cell surface receptors (9). In the reproductive tract, OPN, which is expressed by secretory-phase endometrial cells, invading trophoblasts, decidual glands and the placenta, is temporally involved in blastocyst invasion and placentation $(10,11)$. 
Vascularendothelial growth factor (VEGF) is an endothelial cell-specific mitogen in vitro and is known to be the key factor responsible for vasculogenesis and angiogenesis in a variety of models (12). In rodents, VEGF acts in embryo-endometrium interactions by regulating endometrial vascular permeability and endothelial cell proliferation at implantation sites $(13,14)$. Furthermore, VEGF receptor 1 (VEGFR1) and VEGFR2 have been observed in microvessels during the midsecretory period, emphasizing a possible association between the increased microvascular density (MVD) and vascular permeability (15). These studies have determined two basic roles for VEGF in endometrial tissue: a) The regulation of endometrial vascularization and vascular permeability; b) the establishment of a receptive endometrium to support blastocyst implantation and trophoblast invasion.

Assisted reproductive technology (ART) is now available to select high-quality embryos; however, despite such technological advances, implantation rates remain relatively low (16). Uterine receptivity has a key role in the establishment of successful pregnancies, and impaired receptivity may limit the success of ART. Controlled ovarian hyperstimulation $(\mathrm{COH})$ is a frequently used therapeutic strategy in the treatment of infertility; however, the effect of $\mathrm{COH}$ on implantation remains controversial (17-19). The use of gonadotropin-releasing hormone agonist ( $\mathrm{GnRHa}$ ) is associated with advanced endometrial maturation of 2-4 days on the day of oocyte retrieval, and no pregnancy occurs when the advancement is $>3$ days (20). It has additionally been suggested that $\mathrm{COH}$ results in supraphysiological levels of estrogen and progesterone, which may impair endometrial receptivity (21).

The use of herbal medicines for the treatment of infertility has been well documented in China for numerous years. In contrast to target-oriented Western medicine, Traditional Chinese Medicine (TCM) uses a holistic and synergistic approach to restore the balance of body energy and to maintain the normal functioning of the body $(22,23)$. According to the theory of TCM, we have developed Zi Dan Yin (ZDY) (Table I) to be used to prepare the endometrium for implantation.

Although there have been marked increased in the understanding of implantation, therapeutic options remain poor. Further studies are required to provide clinical treatment options for patients experiencing implantation failure-related infertility. The incidence of early pregnancy loss during or immediately subsequent to implantation is high (25-40\%) (24). Failed implantation is also a major limiting factor in assisted reproduction (25). The aim of the present study was to investigate the effects of $\mathrm{COH}$ on implantation biology and pregnancy outcome, and to explore the potential therapeutic role of the TCM Zi Dan Yin (ZDY).

\section{Materials and methods}

Ethics statement. All of the experimental protocols utilized in the present study were approved by the Ethics Committee of the Beijing University of Chinese Medicine Animal Care and Use Committee (no. 2013-015-A).

ZDY preparation. All the crude drugs involved in the composition of ZDY were obtained from the Department of Pharmacy,
Table I. Composition of Zi Dan Yin.

Components

Ratio

Sheng Di [Rehmannia glutinosa (Gaertn.)

15

Libosch., root)]

Dan Shen (Salviae miltiorrhizae Bge., root)

Dang gui [Angelica sinensis (Oliv.) Diels., root]

Chuan Duan (Dipsacus asperoides C.Y. Cheng

et T.M. Ai., root)

Du Zhong (Eucommia ulmoides Oliv., cortex)

Shan Yao (Dioscorea opposita Thunb., rhizome)

Mei Gui-hua (Rosa rugosa Thunb., flower)

Chuan Xiong (Ligusticum Chuanxiong Hort., rhizome)

Yi Yi-ren [Coix lacryma-jobi L. var. ma-yuen

(Roman.) Stapf., seed]

Dongfang Hospital of Beijing University of Chinese Medicine (Beijing, China). The quality of the raw herbs was controlled according to the requirement of the Pharmacopoeia of the People's Republic of China (26). Aqueous extract of ZDY was prepared in accordance with the following procedure. In brief, nine medicinal materials were mixed in proportion and macerated for $1 \mathrm{~h}$ with eight volumes of distilled water and then decocted for $2 \mathrm{~h}$. The cooled extract was subsequently filtered. The extraction procedure was repeated twice. The extracts were then combined and concentrated by boiling to a final volume of $100 \mathrm{ml}(4.12 \mathrm{~g} / \mathrm{ml})$. This dilution was used in the following preliminary experiments in a range of concentrations from 1.03 to $4.12 \mathrm{~g} / \mathrm{ml}$.

Treatment. Mature, female Sprague Dawley rat virgins aged seven to eight weeks (weight, 200-220 g) were maintained in the Research Centre, Beijing University of Chinese Medicine on a 12-h light/dark regimen with free access to water and a standard diet. The estrous cycle was identified by vaginal smear. Only the female rats with regular cycles were used. Suitable rats were randomly allocated into one of four groups: Control, $\mathrm{COH}, \mathrm{ZDY}$ and $\mathrm{COH}+\mathrm{ZDY}$.

Rats in the $\mathrm{COH}$ group were administered $1 \mathrm{ml} / 100 \mathrm{~g}$ distilled water for 12 days and then treated with the GnRHa-long protocol. In brief, the GnRHa (Diphereline ${ }^{\mathrm{TM}}$; Ipsen, Boulogne-Billancourt, France) was injected intraperitoneally at $1.5 \mu \mathrm{g} / 100 \mathrm{~g}$ body weight/day between days 3 and 9 of the estrous cycle. Pregnant mare's serum gonadotropin (Inner Mongolia Chifeng Bo En Pharmaceutical Co., Ltd., Chifeng, China) was injected intraperitoneally at $5 \mathrm{IU} / 100 \mathrm{~g}$ body weight on the ninth day of the estrous cycle followed by the injection of human chorionic gonadotropin (hCG; Yantai North Pharmaceutical Co., Ltd., Yantai, China) at 10 IU/100 g $28 \mathrm{~h}$ later. In the ZDY group, the animals were administered $1 \mathrm{ml} \mathrm{ZDY} / 100 \mathrm{~g}$ body weight/day for 12 days followed by saline injections at the same time and volume as the $\mathrm{COH}$ group. In the $\mathrm{COH}+\mathrm{ZDY}$ group, the animals were administered the $1 \mathrm{ml} \mathrm{ZDY} / 100 \mathrm{~g}$ daily for 12 days and were then subjected to the same GnRHa-long protocol as the $\mathrm{COH}$ group. In the 
control group, the rats were administered distilled water for 12 days, followed by injections with saline at the same time and volume as the treatments used in the $\mathrm{COH}$ group. The female rats were housed overnight with males (1:1) subsequent to being given hCG or saline. Successful mating was checked daily by the presence of vaginal plugs. The morning when the plug was found was designated the day 1 of gestation (D1).

In rats, implantation occurs on the fifth day after coitus. During this specific, temporary period, known as the 'receptive phase' and 'window of implantation', the endometrium undergoes marked changes in structure and function induced by ovarian steroids, which prepares the endometrium to be receptive to the embryo (5). Thus, to demonstrate the characteristics of the endometrium in a $\mathrm{COH}$ rat model during implantation, the rats were sacrificed using $10 \%$ chloral hydrate (302-17-0; Solabrio, Beijing, China) prior to implantation (D3 and D4) and during the implantation window (D5) ( $n=6 /$ day). Whole uteri were collected promptly without excess fat and connective tissue. One-third of each sample was fixed in $4 \%$ paraformaldehyde, and the remaining part of each sample was stored at $-80^{\circ} \mathrm{C}$ until protein and mRNA extraction.

MVD. For the immunohistochemical detection of cluster of differentiation (CD) 34, uteri were fixed for $12 \mathrm{~h}$ at $4^{\circ} \mathrm{C}$ in buffered paraformaldehyde and were then routinely processed for paraffin embedding. The paraffin-fixed tissues were cut into $4-\mu \mathrm{m}$ sections. Briefly, the slides were dewaxed, rehydrated, blocked and incubated with the primary antibody: Anti-CD34 goat polyclonal antibody (cat. no. sc-1336; Santa Cruz Biotechnology, Inc., Santa Cruz, CA, USA) at a 1:200 dilution overnight at $4^{\circ} \mathrm{C}$. Subsequent to rinsing three times with phosphate-buffered saline (PBS), the tissues were incubated with secondary antibody (PV-0003; ZSGB-BIO, Beijing, China) for $25 \mathrm{~min}$, followed by incubation with a 3,3'-diaminobenzidine kit (ZLI-9018; ZSGB-BIO, Beijing, China). As a control, normal PBS was used and the primary antibody was omitted. Finally, the sections were dehydrated, counterstained and mounted for observation.

MVDs were viewed at x400 magnification (40X objective lens and 10X ocular lens; $0.24 \mathrm{~mm}^{2} /$ field). Tissue images were captured with a digital camera (Olympus, Inc., Tokyo, Japan). For each section, at least five randomly selected fields were counted to determine the density of the microvessels within the uterus. The number of CD34-positive vessels was quantified using Diagnostic Instruments SPOT imaging software (Diagnostic Instruments, Inc., Sterling Heights, MI, USA). The MVD was calculated as the number of CD34-positive vessels/(40x0.24 $\left.\mathrm{mm}^{2}\right)$.

Western blot analysis. Uterine slices previously frozen at $-80^{\circ} \mathrm{C}$ were incubated and lysed in radioimmunoprecipitation assay lysis buffer (cat. no. C1053; Applygen Technologies, Inc., Beijing, China) supplemented with protease inhibitor cocktail (cat. no. P1265; Applygen Technologies, Inc.). The protein concentration was quantified using a bicinchoninic acid assay (cat. no. P1511; Applygen Technologies, Inc.). Sodium dodecyl sulfate-polyacrylamide gel electrophoresis (SDS-PAGE) using a $10 \%$ polyacrylamide gel was performed and the samples were transferred to nitrocellulose membranes (Bio-Rad, Hercules, CA, USA). The membranes were blotted with rabbit polyclonal anti-VEGF (cat. no. ab46154; Abcam, Cambridge, UK) or rabbit polyclonal anti-OPN (cat. no. ab8448; Abcam) primary antibodies at a dilution of 1:2,000 and incubated overnight at $4^{\circ} \mathrm{C}$. Following incubation, the membranes were washed three times with Tris-buffered saline/Tween 20 and then incubated with goat anti-rabbit immunoglobulin G secondary antibodies (cat. nos. P1308 and P1309; Applygen Technologies, Inc.) at a dilution of 1:2,000 at room temperature for $1 \mathrm{~h}$. The blots were visualized with Super ECL Plus Detection Reagent (cat. no. P1010; Applygen Technologies, Inc.). The enhanced chemiluminescence signals were detected with Quantity One ${ }^{\circledR}$ software (Bio-Rad). $\beta$-actin (cat. no. ab8226; Abcam) was used as the reference protein to validate the amount of protein loaded onto the gel.

Reverse transcription-quantitative polymerase chain reaction (RT-qPCR). VEGF and OPN gene expression was validated using qPCR. Total RNA was extracted from the uteri of rats in the control, $\mathrm{COH}, \mathrm{ZDY}$ and $\mathrm{COH}+\mathrm{ZDY}$ groups using TRIzol ${ }^{\circledR}$ (Invitrogen Life Technologies, Carlsbad, CA, USA) according to the manufacturer's instructions. RNA was thawed on ice and quantified spectrophotometrically, and the quality was then assessed using SDS-PAGE. RT was performed with $8 \mu$ l total RNA per $20 \mu \mathrm{l}$ reaction using the standard cDNA Synthesis kit (Takara Bio, Inc., Shiga, Japan). The qPCR primer sequences for the target genes were as follows: GAPDH forward primer, 5'-TGC TGA GTA TGT CGT GGA G-3' and reverse primer, 5'-GTC TTC TGA GTG GCA GTG AT- 3' (288 bp); VEGF forward primer, 5'-GGC TCA CTT CCA GAA ACA CG-3' and reverse primer, 5'-GTG CTC TTG CAG AAT CTA GTG G-3' (165 bp); OPN forward primer, 5'-GAG GTG ATA GCT TGG CTT ACG G-3' and reverse primer, 5'-CGC TGG GCA ACT GGG ATG-3' (154 bp).

For each qPCR reaction, the typical thermal cycling conditions included an initial activation step at $95^{\circ} \mathrm{C}$ for $5 \mathrm{~min}$, 40 cycles of amplification and a final melting curve $\left(65-95^{\circ} \mathrm{C}\right)$. PCR reactions were performed using an ABI Prism ${ }^{\circledR} 7700$ Sequence Detection system (Applied Biosystems, Foster City, CA, USA). Experiments were carried out in triplicate, and cDNA concentrations were normalized with the GAPDH PCR products. Gene expression was analyzed using the $2^{-\Delta \Delta C t}$ algorithm.

Average number of implantation sites and live births. On D10, uteri were collected from each group immediately subsequent to sacrifice $(n=6)$. Whole uteri were collected promptly without excess fat and connective tissue, and the conceptuses were removed from the uteri. The number of implantation sites in the uterine horn was recorded. The average number of implantation sites was calculated as the total number of implantation sites/number of rats. Subsequent to conceiving and bearing newborn rats, another sample of rats $(n=6)$ were used to conceive and bear newborn rats to determine the number of live births from each group. The average number of live births was calculated as the total number of newborn rats/number of rats.

Statistical analysis. Data are presented as the mean \pm standard error of the mean. One-way analysis of variance and least significant difference tests were used with the SPSS 17.0 
Table II. Microvascular density.

\begin{tabular}{lccr}
\hline Group & D3 (n) & D4 (n) & D5 (n) \\
\hline Control & $1.79 \pm 0.06$ & $2.88 \pm 0.19$ & $3.56 \pm 0.11$ \\
COH & $2.24 \pm 0.09^{\mathrm{a}}$ & $3.91 \pm 0.07^{\mathrm{a}}$ & $4.34 \pm 0.11^{\mathrm{a}}$ \\
ZDY & $1.86 \pm 0.03$ & $2.88 \pm 0.09$ & $3.34 \pm 0.10$ \\
COH + ZDY & $1.74 \pm 0.07$ & $2.97 \pm 0.08$ & $3.32 \pm 0.09$ \\
\hline
\end{tabular}

The density of microvessels within the uterus was determined on D3, D4 and D5. Data are presented as the mean \pm standard error of the mean ( $\mathrm{n}=6$ in each group). ${ }^{\mathrm{a}} \mathrm{P}<0.05$ vs. all other groups. No significant differences were found among the control, ZDY and COH $+\mathrm{ZDY}$ groups . The groups were established as follows: $\mathrm{COH}$, GnRHa-long protocol-stimulated rats; ZDY, rats that received ZDY treatment; COH + ZDY, GnRHa long protocol-stimulated rats that received ZDY treatment. $\mathrm{COH}$, controlled ovarian hyperstimulation; ZDY, Zi Dan Yin; D3, day 3 of pregnancy; GnRHa, gonadotrophin-releasing hormone agonist.

Table III. Number of implantation sites and live births.

\begin{tabular}{lcccc}
\hline Parameter & Control & COH & ZDY & COH + ZDY \\
\hline Implantation sites (n) & $10.33 \pm 0.84$ & $3.67 \pm 0.49^{\mathrm{a}}$ & $10.00 \pm 0.73$ & $8.67 \pm 0.33$ \\
Live births (n) & $11.00 \pm 0.63$ & $4.33 \pm 0.42^{\mathrm{a}}$ & $10.67 \pm 0.71$ & $9.33 \pm 0.61$ \\
\hline
\end{tabular}

Data are presented as the mean \pm standard error of the mean $\left(n=6\right.$ in each group). ${ }^{\mathrm{a}} \mathrm{P}<0.05$ vs. all other groups. No significant differences were found among the control, ZDY and COH + ZDY groups. The groups were established as follows: $\mathrm{COH}$, GnRHa long protocol-stimulated rats; ZDY, rats that received ZDY treatment; $\mathrm{COH}+\mathrm{ZDY}$, GnRHa-long protocol-stimulated rats that received ZDY treatment. $\mathrm{COH}$, controlled ovarian hyperstimulation; ZDY, Zi Dan Yin; D3, day 3 of pregnancy; GnRHA, gonadotrophin-releasing hormone agonist.

statistical software package (SPSS, Inc., Chicago, IL, USA). $\mathrm{P}<0.05$ was considered to indicate a statistically significant difference. Graphs of the data were produced using Microsoft Excel software (Microsoft Corp., Redmond, WA, USA).

\section{Results}

MVD results. A summary of the MVD results is shown in Table II. Through the immunohistochemical analysis, the MVD was found to be significantly increased in the $\mathrm{COH}$ group compared with that in the control, ZDY and $\mathrm{COH}+\mathrm{ZDY}$ groups $(\mathrm{P}<0.01)$, with a maximal value on $\mathrm{D} 5$. No significant differences were found among the control, ZDY and $\mathrm{COH}+\mathrm{ZDY}$ groups $(\mathrm{P}>0.05)$.

Western blot analysis results. The protein expression of endometrial VEGF and OPN during implantation was confirmed by western blotting (Fig. 1). The protein levels of VEGF and OPN were normalized by $\beta$-actin. Among the four groups, the VEGF and OPN expression levels in the $\mathrm{COH}$ group were significantly higher than those in the other groups on D3, D4 and D5 $(\mathrm{P}<0.05)$. No significant differences were found among the control, ZDY and $\mathrm{COH}+\mathrm{ZDY}$ groups $(\mathrm{P}>0.05)$.

RT- $q$ PCR results. To demonstrate whether the VEGF and OPN mRNA expression results were consistent with the results of protein expression, RT-qPCR analysis was used (Fig. 2). VEGF and OPN mRNA were found to be expressed in the endometrium of the rats during the implantation window. Compared with the control, ZDY and COH + ZDY groups, the VEGF and
OPN mRNA expression levels, normalized by GAPDH, in the $\mathrm{COH}$ group were significantly increased $(\mathrm{P}<0.05)$. During the implantation window, no significant differences were found among the control, ZDY and $\mathrm{COH}+\mathrm{ZDY}$ groups $(\mathrm{P}>0.05)$.

Measurements of the number of implantation sites and live births. The effects of $\mathrm{COH}$ and/or ZDY treatment on the number of implantation sites and live births are summarized in Table III. The number of implantation sites and live births in the $\mathrm{COH}$ group was significantly lower than that in the other groups. No significant differences were found among the control, ZDY and $\mathrm{COH}+\mathrm{ZDY}$ groups.

\section{Discussion}

The early stages of embryo implantation in rats are characterized by two endometrial vascular events: A localized increase in endometrial vascular permeability, which is the earliest indicator of the implantation, and increased endothelial cell proliferation (13). VEGF exhibits a potent vascular permeability-inducing action and has been revealed to be the factor responsible for implantation-related increases in vascular permeability in the rat endometrium. Furthermore, the role of VEGF in vascular beds has been demonstrated in other mammalian species, including humans, pigs and rabbits $(27,28)$. A previous study showed that endothelial cell proliferation was increased from the third day of pregnancy and remained elevated throughout the entire endometrium up to the fifth day of pregnancy (29). The present study indicated that VEGF can be found in the rat endometrium on D3, D4 and D5. 
A

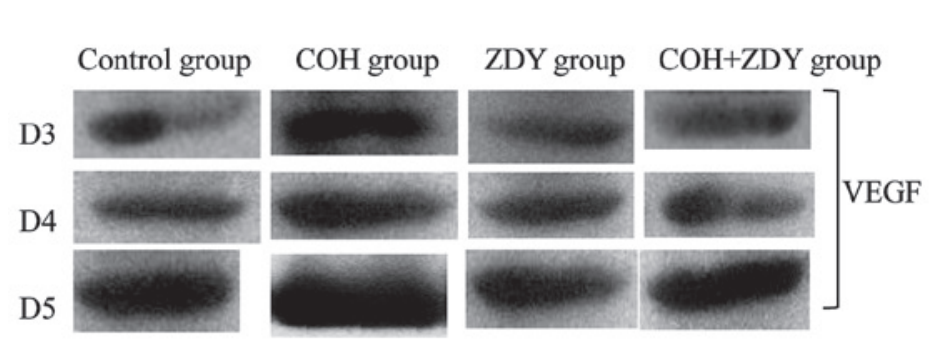

D3

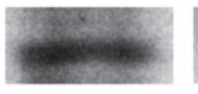

D4

D5
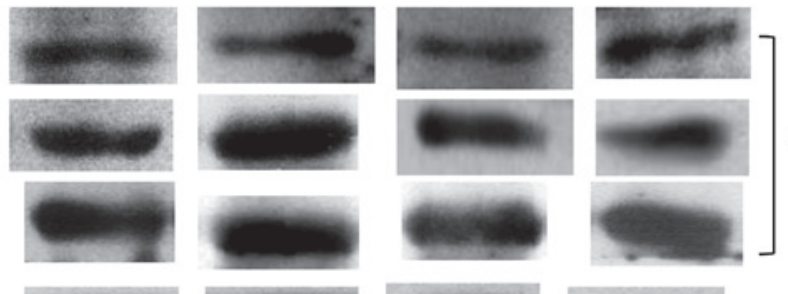

Can
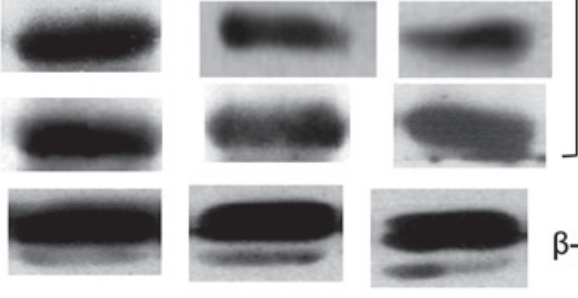

(1)

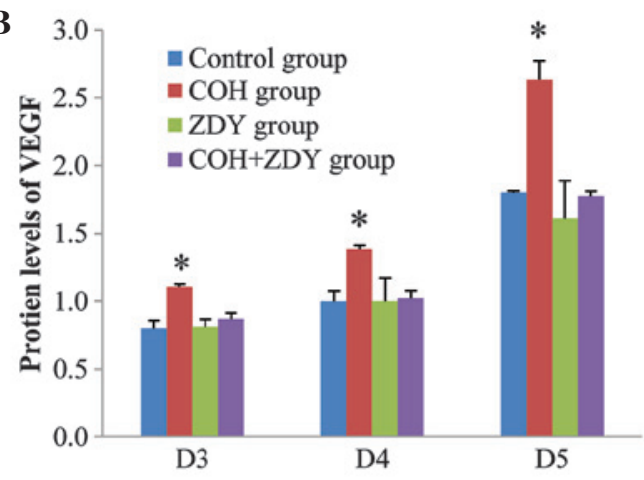

C

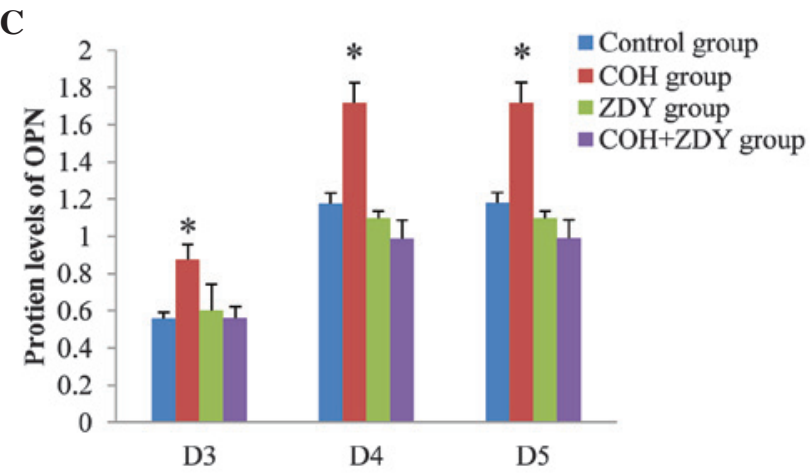

Figure 1. Protein expression of VEGF and OPN. (A) Western blotting was used to measure VEGF and OPN protein levels in COH rats receiving different treatments and controls. (B) VEGF and (C) OPN protein expression in the COH group was increased compared with that in the control, ZDY and COH + ZDY groups. No significant differences were found among the control, ZDY and COH + ZDY groups $(n=6)$. Data are expressed as the mean \pm standard error of the mean. "P<0.05 vs. all other groups. VEGF, vascular endothelial growth factor; OPN, osteopontin; COH, controlled ovarian hyperstimulation; ZDY, Zi Dan Yin; D3, day 3 of pregnancy.
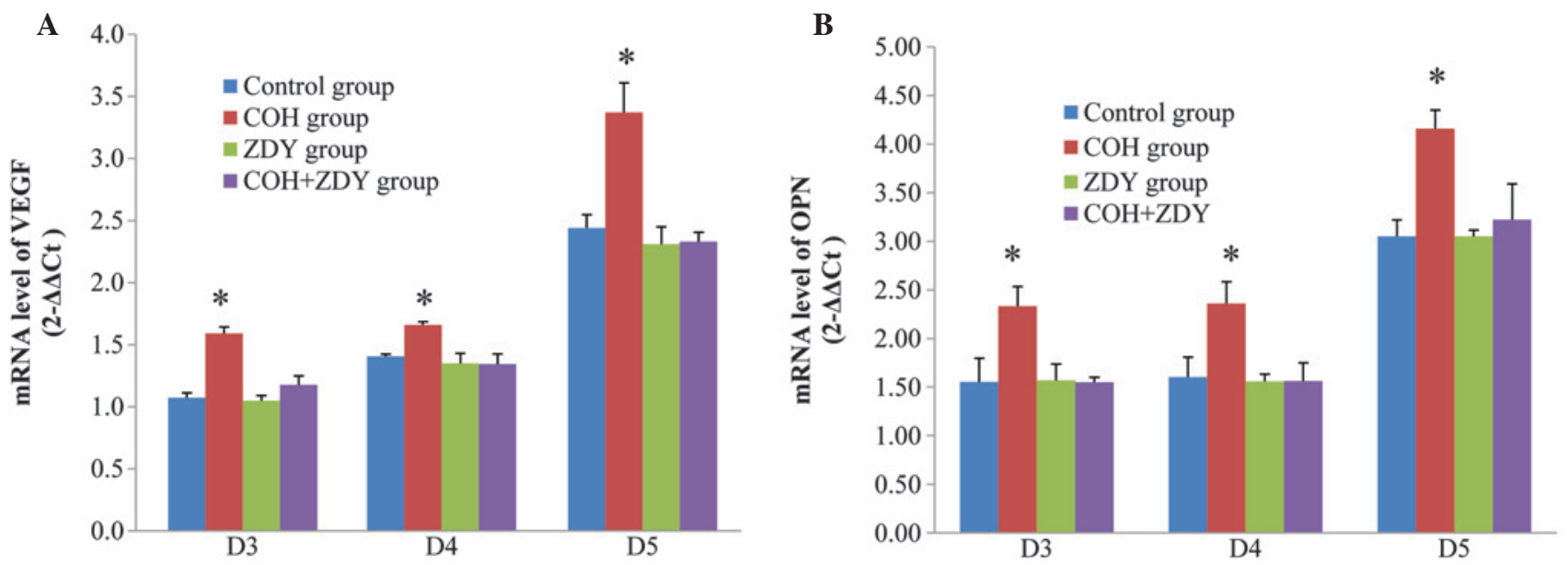

Figure 2. mRNA expression of VEGF and OPN. (A) VEGF and (B) OPN mRNA expression in different groups. VEGF and OPN mRNA expression in the $\mathrm{COH}$ group was increased compared with that in the control, ZDY and COH + ZDY groups. No significant differences were found among the control, ZDY and $\mathrm{COH}+\mathrm{ZDY}$ groups $(\mathrm{n}=6)$. Data are expressed as the mean \pm standard error of the mean. " $\mathrm{P}<0.05$ vs. all other groups. VEGF, vascular endothelial growth factor; OPN, osteopontin; $\mathrm{COH}$, controlled ovarian hyperstimulation; ZDY, Zi Dan Yin; D3, day 3 of pregnancy.

One of the commonest methods of indirectly assessing vasculogenic and angiogenic activity is to examine and compare the net gain or loss in the number of microvessels between different tissues. This is most frequently used to count the number of vessel cross-sections or profiles present in a specified area of a histological section. Following the invasion of the maternal endometrium, embryonic development is characterized by a marked growth of blood vessels occurring concurrently with decidualization and the development of vascular membranes (30). Previous studies have demonstrated the presence of VEGF mRNA in trophoblast cells in rats (31) and mice (32). These findings suggest that VEGF may be a key factor in the induction of vascular growth in the decidua and vascular membranes; however, there is little knowledge concerning the direct association between the MVD and VEGF expression. To the best of our knowledge, this study 
is the first to measure the MVD, as well as VEGF expression, in a $\mathrm{COH}$ rat model. The findings showed that the changes in MVD and VEGF expression are consistent in the rat endometrium throughout implantation. Furthermore, OPN showed the same expression trend at the same time. We suggest the existence of an association between the MVD and the levels of VEGF and OPN.

Implantation is a complex process involving proliferation and tissue remodeling. Adhesion molecules and cytokines have been suggested to play key roles in this process (33), and OPN has the capacity to act as both a cytokine and an adhesion molecule. A previous study has shown that OPN expression is increased in the secretory-phase endometrium and decidua of pregnant females (34). Furthermore, microarray studies have consistently found a 4.9- to 20-fold upregulation of OPN during this period in humans (33-38). In mice, OPN induces blastocysts to activate their adhesion competence through the formation of integrin adhesion complexes at the trophectoderm cell surface (39). By contrast, OPN-null mice remain fertile $(40,41)$. In the present study, OPN protein levels were low on D3 and D4 and markedly increased on D5. OPN mRNA levels were low on D3, and markedly increased on D4 and D5. OPN expression therefore increased throughout the implantation process. It has been suggested that angiogenesis and vascular remodeling is facilitated by OPN and $\alpha_{v} \beta_{3}(42)$. This is one possible explanation for the increased OPN expression associated with increased MVD.

$\mathrm{COH}$ was observed to have a negative effect on the endometrium, as the $\mathrm{COH}$ rat model exhibited a significant higher MVD and VEGF and OPN expression compared with the control group rats. Previous studies have reported increased pre-implantation mortality following superovulation in mice $(43,44)$, hamsters $(45)$ and rats $(46)$. Furthermore, impaired development and reduced implantation rates were found with embryos from superovulated donors, although the weight of the live fetuses obtained following transfer from superovulated donors was not significantly different from that of control embryos; this may suggest that superovulation has certain negative effects even on viable embryos (47). Failed implantation is a significant limiting factor in assisted reproduction (25). Since ovarian stimulation generates a cascade of hormonal and physiological events, the embryos mature in an environment that exhibits differences from the environment in which embryos mature naturally (48). Furthermore, variations may also be apparent in the timing of ovulation and implantation (49). The relative contribution of the endometrium to the rate of successful reception is not currently known, and no accepted criteria exist for the evaluation of endometrial implantation; however, preparations of the endometrium-produced cytokines may mediate these precisely defined morphological changes $(21,50)$. In the present study, the expression of VEGF and OPN in the $\mathrm{COH}$ group was higher than that in the control, ZDY and $\mathrm{COH}+\mathrm{ZDY}$ groups, indicating that $\mathrm{COH}$ may impair the synchronization of embryonic development and endometrial maturation; however, the adverse effects of $\mathrm{COH}$ cannot only be attributed to asynchrony, as an early study showed that superovulation following synchronization also resulted in increased embryonic loss (43).

In the present study, ZDY suppressed the $\mathrm{COH}$-induced increases in MVD and VEGF and OPN expression in rats.
No significant differences were found between the control and ZDY groups. These results therefore suggest that a TCM such as ZDY could prove to be of clinical use in patients with impaired endometrial receptivity subsequent to $\mathrm{COH}$; however, TCM cannot translate the normal state into a super-normal state, which accounts for a lack of significant difference between the control and ZDY groups. A further clinical study is required to confirm the proposed approach in this aspect.

In combination, the present results indicated that $\mathrm{COH}$ treatment increased the expression of MVD, VEGF and OPN during implantation. The study showed that VEGF and OPN may play important roles during implantation, and that an association exists between changes in the MVD and VEGF and OPN expression. In addition to the molecular and structural markers of endometrial receptivity, MVD could be an alternative approach to identify this period of receptivity in rats. The present study also revealed that high levels of VEGF and OPN were apparent subsequent to $\mathrm{COH}$ treatment. These findings confirmed the adverse effects of $\mathrm{COH}$ with $\mathrm{GnRHa}$ on implantation, which have been documented in previous studies (47,51). ZDY markedly restored the endometrial MVD and VEGF and OPN expression during implantation in the $\mathrm{COH}$ rat model. The present study provides novel insight into TCM approaches for infertility treatment and ART.

\section{Acknowledgements}

This study was supported by the Natural Science Foundation of China (no. 81173292).

\section{References}

1. Finn CA and Martin L: The control of implantation. J Reprod Fertil 39: 195-206, 1974.

2. Tabibzadeh S and Babaknia A: The signals and molecular pathways involved in implantation, a symbiotic interaction between blastocyst and endometrium involving adhesion and tissue invasion. Hum Reprod 10: 1579-1602, 1995.

3. Cakmak $\mathrm{H}$ and Taylor HS: Implantation failure: molecular mechanisms and clinical treatment. Hum Reprod Update 17: 242-253, 2011.

4. Sharkey AM and Smith SK: The endometrium as a cause of implantation failure. Best Pract Res Clin Obstet Gynaecol 17: 289-307, 2003.

5. Psychoyos A: Hormonal control of ovoimplantation. Vitam Horm 31: 201-256, 1973.

6. Psychoyos A: Hormonal control of uterine receptivity for nidation. J Reprod Fertil Suppl: 17-28, 1976.

7. Renfree MB and Shaw G: Diapause. Annu Rev Physiol 62: 353-375, 2000.

8. Oldberg A, Franzén A and Heinegård D: Cloning and sequence analysis of rat bone sialoprotein (osteopontin) cDNA reveals an Arg-Gly-Asp cell-binding sequence. Proc Natl Acad Sci USA 83: 8819-8823, 1986

9. Ruoslahti E and Pierschbacher MD: New perspectives in cell adhesion: RGD and integrins. Science 238: 491-497, 1987.

10. Brown LF, Berse B, Van de Water L, et al: Expression and distribution of osteopontin in human tissues: widespread association with luminal epithelial surfaces. Mol Biol Cell 3: 1169-1180, 1992.

11. Nomura S, Wills AJ, Edwards DR, Heath JK and Hogan BL: Developmental expression of 2 ar (osteopontin) and SPARC (osteonectin) RNA as revealed by in situ hybridization. J Cell Biol 106: 441-450, 1988.

12. Maglione D, Guerriero V, Viglietto G, et al: Two alternative mRNAs coding for the angiogenic factor, placenta growth factor (PlGF), are transcribed from a single gene of chromosome 14. Oncogene 8: 925-931, 1993 
13. Rabbani M and Rogers PA: Role of vascular endothelial growth factor in endometrial vascular events before implantation in rats. Reproduction 122: 85-90, 2001.

14. Rockwell LC, Pillai S, Olson CE and Koos RD: Inhibition of vascular endothelial growth factor/vascular permeability factor action blocks estrogen-induced uterine edema and implantation in rodents. Biol Reprod 67: 1804-1810, 2002.

15. Krüssel JS, Casañ EM, Raga F, et al: Expression of mRNA for vascular endothelial growth factor transmembraneous receptors Flt1 and KDR, and the soluble recetor sflt in cycling human endometrium. Mol Hum Reprod 5: 452-458, 1999.

16. de Mouzon J, Goossens V, Bhattacharya S, et al; European IVF-monitoring (EIM) Consortium, for the European Society of Human Reproduction and Embryology (ESHRE): Assisted reproductive technology in Europe, 2006: results generated from European registers by ESHRE. Hum Reprod 25: 1851-1862, 2010.

17. Levi AJ, Drews MR, Bergh PA, Miller BT and Scott RT Jr: Controlled ovarian hyperstimulation does not adversely affect endometrial receptivity in in vitro fertilization cycles. Fertil Steril 76: 670-674, 2001

18. Pattinson HA, Greene CA, Fleetham J and Anderson-Sykes SJ: Exogenous control of the cycle simplifies thawed embryo transfer and results in a pregnancy rate similar to that for natural cycles. Fertil Steril 58: 627-629, 1992

19. Pellicer A, Valbuena D, Cano F, Remohí J and Simón C: Lower implantation rates in high responders: evidence for an altered endocrine milieu during the preimplantation period. Fertil Steril 65: 1190-1195, 1996

20. Bourgain $C$ and Devroey P: The endometrium in stimulated cycles for IVF. Hum Reprod Update 9: 515-522, 2003.

21. Dey SK, Lim H, Das SK, et al: Molecular cues to implantation. Endocr Rev 25: 341-373, 2004

22. Efferth T, Li PC, Konkimalla VSB and Kaina B: From traditional Chinese medicine to rational cancer therapy. Trends Mol Med 13: 353-361, 2007.

23. Wen Z, Wang Z, Wang S, et al: Discovery of molecular mechanisms of traditional Chinese medicinal formula $\mathrm{Si}-\mathrm{Wu}$-Tang using gene expression microarray and connectivity map. PLoS One 6: e18278, 2011

24. Wilcox AJ, Weinberg CR, O'Connor JF, et al: Incidence of early loss of pregnancy. New Engl J Med 319: 189-194, 1988.

25. Norwitz ER, Schust DJ and Fisher SJ: Implantation and the survival of early pregnancy. N Engl J Med 345: 1400-1408, 2001.

26. Zhong-zhi Q, Yang D, Yan-ze L, et al: Pharmacopoeia of the People's Republic of China (2010 Edition): A Milestone in Development of China's Healthcare. Chinese Herbal Medicines 2: 157-160, 2010.

27. Collins PD, Connolly DT and Williams TJ: Characterization of the increase in vascular permeability induced by vascular permeability factor in vivo. Br J Pharmacol 109: 195-199, 1993

28. Hippenstiel S, Krüll M, Ikemann A, et al: VEGF induces hyperpermeability by a direct action on endothelial cells. Am J Physiol 274: L678-L684, 1998.

29. Goodger AM and Rogers PA: Uterine endothelial cell proliferation before and after embryo implantation in rats. J Reprod Fertil 99: 451-457, 1993.

30. Ramsey EM and Donner MW: Placental Vasculature and Circulation: Anatomy, Physiology, Radiology, Clinical Aspects: Atlas and Textbook. Thieme, Stuttgart, 1980.

31. Jakeman LB, Armanini M, Phillips HS and Ferrara N: Developmental expression of binding sites and messenger ribonucleic acid for vascular endothelial growth factor suggests a role for this protein in vasculogenesis and angiogenesis. Endocrinology 133: 848-859, 1993.
32. Breier G, Albrecht U, Sterrer S and Risau W: Expression of vascular endothelial growth factor during embryonic angiogenesis and endothelial cell differentiation. Development 114: 521-532, 1992.

33. Giudice LC: Genes associated with embryonic attachment and implantation and the role of progesterone. J Reprod Med 44 (2 Suppl): 165-171, 1999.

34. Young MF, Kerr JM, Termine JD, et al: cDNA cloning, mRNA distribution and heterogeneity, chromosomal location, and RFLP analysis of human osteopontin (OPN). Genomics 7: 491-502, 1990

35. Carson DD, Lagow E, Thathiah A, et al: Changes in gene expression during the early to mid-luteal (receptive phase) transition in human endometrium detected by high-density microarray screening. Mol Hum Reprod 8: 871-879, 2002.

36. KaoL, Tulac S,LoboSA, et al: Global gene profiling in human endometrium during the window of implantation. Endocrinology 143: 2119-2138, 2002

37. Borthwick JM, Charnock-Jones DS, Tom BD, et al: Determination of the transcript profile of human endometrium. Mol Hum Reprod 9: 19-33, 2003.

38. Talbi S, Hamilton A, Vo K, et al: Molecular phenotyping of human endometrium distinguishes menstrual cycle phases and underlying biological processes in normo-ovulatory women. Endocrinology 147: 1097-1121, 2006.

39. Chaen T, Konno T, Egashira M, et al: Estrogen-dependent uterine secretion of osteopontin activates blastocyst adhesion competence. PLoS One 7: e48933, 2012.

40. Liaw L, Birk DE, Ballas CB, Whitsitt JS, Davidson JM and Hogan BL: Altered wound healing in mice lacking a functional osteopontin gene (spp1). J Clin Invest 101: 1468-1478, 1998.

41. Rittling SR, Matsumoto HN, Mckee MD, et al: Mice lacking osteopontin show normal development and bone structure but display altered osteoclast formation in vitro. J Bone Miner Res 13: 1101-1111, 1998.

42. Liaw L, Almeida M, Hart CE, Schwartz SM and Giachelli CM: Osteopontin promotes vascular cell adhesion and spreading and is chemotactic for smooth muscle cells in vitro. Circ Res 74: 214-224, 1994.

43. Beaumont HM and Smith AF: Embryonic mortality during the pre- and post-implantation periods of pregnancy in mature mice after superovulation. J Reprod Fertil 45: 437-448, 1975.

44. Ertzeid G and Storeng R: Adverse effects of gonadotrophin treatment on pre- and postimplantation development in mice. J Reprod Fertil 96: 649-655, 1992.

45. McKiernan SH and Bavister BD: Gonadotrophin stimulation of donor females decreases post-implantation viability of cultured one-cell hamster embryos. Hum Reprod 13: 724-729, 1998

46. Miller BG and Armstrong DT: Effects of a superovulatory dose of pregnant mare serum gonadotropin on ovarian function, serum estradiol, and progesterone levels and early embryo development in immature rats. Biol Reprod 25: 261-271, 1981.

47. Ertzeid G and Storeng R: The impact of ovarian stimulation on implantation and fetal development in mice. Hum Reprod 16: 221-225, 2001.

48. Foote R and Ellington J: Is a superovulated oocyte normal? Theriogenology 29: 111-123, 1988.

49. Allen J and McLaren A: Cleavage rate of mouse eggs from induced and spontaneous ovulation. J Reprod Fertil 27: 137-140, 1971.

50. Makrigiannakis A, Minas V, Kalantaridou SN, Nikas G and Chrousos GP: Hormonal and cytokine regulation of early implantation. Trends Endocrinol Metab 17: 178-185, 2006.

51. Ruan HC, Zhu XM, Luo Q, et al: Ovarian stimulation with GnRH agonist, but not GnRH antagonist, partially restores the expression of endometrial integrin beta 3 and leukaemia-inhibitory factor and improves uterine receptivity in mice. Hum Reprod 21: 2521-2529, 2006. 